\title{
An optimized encoding algorithm for systematic polar codes
}

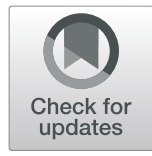

Xiumin Wang ${ }^{1}$, Zhihong Zhang ${ }^{1}$, Jun $\mathrm{Li}^{2^{*}}$, Yu Wang ${ }^{3}$, Haiyan Cao ${ }^{4}$, Zhengquan $\mathrm{Li}^{5}$ and Liang Shan ${ }^{1}$

\begin{abstract}
Many different encoding algorithms for systematic polar codes (SPC) have been introduced since SPC was proposed in 2011. However, the number of the computing units of exclusive OR (XOR) has not been optimized yet. According to an iterative property of the generator matrix and particular lower triangular structure of the matrix, we propose an optimized encoding algorithm (OEA) of SPC that can reduce the number of XOR computing units compared with existing non-recursive algorithms. We also prove that this property of the generator matrix could extend to different code lengths and rates of the polar codes. Through the matrix segmentation and transformation, we obtain a submatrix with all zero elements to save computation resources. The proportion of zero elements in the matrix can reach up to $58.5 \%$ from the OEA for SPC when the code length and code rate are 2048 and 0.5, respectively. Furthermore, the proposed OEA is beneficial to hardware implementation compared with the existing recursive algorithms in which signals are transmitted bidirectionally.
\end{abstract}

Keywords: Encoding algorithm, Low complexity, Computing units, Systematic polar codes, Generator matrix transformation

\section{Introduction}

Polar codes proposed by Arikan [1] can theoretically reach the Shannon limit. It has been widely given attention in the communication field because of its low complexity and good decoding performance. As early as 2008, Arikan proposed the concept of channel polarization and carried out a rigorous mathematical proof [2]. For the selection of information bits of polar codes, there are some patterns, such as Bhattacharyya parameters [3] and density evolution [4]. The hardware implementations of the traditional non-systematic polar code (NSPC) encoding were introduced [57]. However, the complexity and computing resources were significantly consumed when fully utilizing the generator matrix of the polar codes to design the hardware structure. Hoyoung et al. [8] proposed partially parallel encoder structure that can reduce latency. However, the resource consumption increased due to the length of the polar codes. Compared to NSPC, the SPC proposed by Arikan [9] outperformed them in the bit error rate. Vangala et al. [10] proposed three encoding algorithms and

\footnotetext{
* Correspondence: 07a0303105@cjlu.edu.cn

${ }^{2}$ Binjiang College Nanjing University of Information Science and Technology, Wuxi 214105, China

Full list of author information is available at the end of the article
}

used the recursion method to find a suitable equilibrium between a memory cell and a computing unit. Chen et al. [11] improved the encoding algorithms designed by Vangala et al. [10] to simplify the storage patterns for SPC. In [12], Sarkis et al. introduced SPC and developed a hardware structure of the decoder. The improved hardware structure increased the throughput of the polar decoder. When the hardware was implemented [13], the input and output ports were exchanged to obtain the SPC encoder based on the NSPC encoder. However, the latency and resource consumption were twice than that of the hardware implementation with NSPC. Wang et al. found a method that utilized the property of parity check matrix to reduce the computational units [14-16]. This approach can be utilized for both SPC and NSPC to reduce computational units. By studying the encoding flow graph of SPC, we found out that some of computational units can be omitted. This omission is determined by the encoding structure and the exclusive Or (XOR) operations.

In this paper, we define some variables which are determined by code length, code rate, and the selection for information bits. The two lemmas regarding the transformed generator matrix have been proved. After applying the property of the transformed generator 
matrix, the proposed algorithm can reduce the number of XOR computing units according to the iterative property of the generator matrix. The iteration property can make the submatrix with a particular lower triangular structure. This submatrix is a part of the original generator matrix and can have all elements with zero value. Besides the zero submatrices obtained from the iteration process, there are still some other elements with zero value in the original matrix. These extra zero elements can also be omitted when computing XOR logical operations.

The rest sections of this paper are organized as follows. The comparisons between SPC and NSPC are introduced in Section 2. The existing encoding algorithms of SPC are reviewed in Section 3. Our simplified process to use XOR operations is also discussed in this section. The distribution rule of information bits, definition of variables, and the lemma proof are shown in Section 4. The case study of our optimized encoding algorithm (OEA) matrix transformation is presented in Section 5. Results and analysis are discussed in Section 6. Conclusions are found in Section 7.

\subsection{Methods/Experimental}

The research content of this paper is mainly theoretical derivation and analysis, and specific experimental verification will be carried out in future research.

\section{Systemic and non-systemic polar codes}

Based on the channel polarization theory, the length of polar codes is $N=2^{n}, n \geq 1$, where $n$ is a positive integer. Let $A$ represent the set of the indices of the information bits. The code rate is $R=K / N$, where $K$ represents the length of information bits and is the number of the elements in a set of $A$. Information bits selection is determined by Bhattacharyya parameters [17]. Let $A c$ represent the complement of a set of $A$. The index set $A c=\{0,1, \ldots, N-1\}-A$ is for the frozen bits, and the length of $A c$ is $N-K \cdot u=\left(u_{0}, u_{1}, \cdots, u_{N-1}\right)$ represents the message vector, where $u_{i}$ denotes an arbitrary element of the vector of $u . x=\left(x_{0}, x_{1}, \cdots, x_{N-1}\right)$ represents a codeword vector, where $x_{i}$ indicates a random component in the vector of $x$. The generator matrix $G_{N}$ is defined as

$$
G_{N}=F^{\otimes n}
$$

where $\otimes$ denotes Kronecker power operation, $n=$ $\log _{2}(N)$, and $F$ denotes two-dimensional matrix $F=[1$, $0 ; 1,1]$. Applying the property of Kronecker product, we can construct a generator matrix as
$G_{N}=\left[\begin{array}{cc}F^{\otimes n-1} & 0 \\ F^{\otimes n-1} & F^{\otimes n-1}\end{array}\right]=\left[\begin{array}{cc}G_{N / 2} & 0 \\ G_{N / 2} & G_{N / 2}\end{array}\right]$

The codeword vector of $x$ for NSPC can be represented by the encoding Eq. (1)

$x=u G_{N}=u_{A} G_{A}+u_{A c} G_{A c}$

where $G A$ is a submatrix of $G N$, and it is constructed by the rows of indices in $A$. GAc is a submatrix of $G N$ and is constructed by the rows of indices in $A c . u A=$ (ui: $i \in A), u A \quad u$ and $u A c=(u j: j \in A c), u A c \quad u$, and $u A c$ $=u-u A$. The symbol of $\oplus$ represents a mod- 2 addition or a logical XOR operation in the binary domain.

Arikan [9] first proposed the mathematical formula shown in Eqs. (4) and (5) for SPC:

$$
\begin{aligned}
& x_{A}=u_{A} G_{A A}+u_{A c} G_{A c A} \\
& x_{A c}=u_{A} G_{A A c}+u_{A c} G_{A c A c}
\end{aligned}
$$

where $G_{A A}$ denotes the submatrix of $G_{N}$ consisting of the array of elements $\left(G_{i, j}\right)$ with $i \in A, j \in A$, and $G_{A A}=$ $\left(G_{i j}: i \in A, j \in A\right)$. Similarly for the other submatrices of $G_{A c A}=\left(G_{i, j}: i \in A c, j \in A\right), G_{A A c}=\left(G_{i j}: i \in A, j \in A c\right)$, and $G_{A c A c}=\left(G_{i, j}: i \in A c, j \in A c\right)$. There is the same denotation for $x_{A}=\left(x_{i}: i \in A\right)$ and $x_{A c}=\left(x_{j}: j \in A c\right)$. If the matrix $G_{A A}$ is invertible and the inputs to SPC encoder are $u_{A c}$ and $x_{A}$, then the output $x_{A c}$ from the SPC encoder is

$x_{A c}=\left(x_{A}-u_{A c} G_{A c A}\right) G_{A A}^{-1} G_{A A c}+u_{A c} G_{A c A c}$

Consider that the decoding results are not affected by the value of frozen bits [1], we can simplify the encoding procedure by setting zero values to all frozen bits, namely $u_{A c}=\left(u_{i}=0: i \in A c\right)$. Then, $x_{A c}$ in Eq. (6) can be simplified to

$$
x_{A c}=x_{A} G_{A A}^{-1} G_{A A c}
$$

where $G_{A A}{ }^{-1}$ is a lower triangular matrix with ones on the diagonal and the submatrix of $G_{A A c}$ also includes a lower triangular matrix. Hence, the matrix product of $G_{A A}{ }^{-1} G_{A A c}$ has the same structure as $G_{A A c}$. It has a submatrix including a lower triangular matrix. We will use the property of $G_{A A}{ }^{-1}=G_{A A}$ in the binary domain [9] to prove the Lemma 1 in Section 4 and the matrix transformation in Section 5.

\section{Proposed OEA for SPC}

The development of the proposed OEA was motivated by $[9,11]$. The computational complexity can be decreased by reducing the number of logical XOR computing units. The following example illustrates the procedure of omitting XOR computing units. After logical XOR operations, the output results are the same 
either from the approach to apply a generator matrix or the method to apply an encoding diagram scheme.

Figure 1 shows the SPC encoding diagram with $N=8$ and $A=\{1,3,5,6,7\}$. In Fig. 1 , the encoding direction is from bottom to top. The encoding process for information bits starts from right to left. Then, the encoding process for frozen bits is from left to right. The gray circles on the rightmost represent the information bits, and the black circles on the leftmost represent the frozen bits. The black arrow on the right represents the computing direction of the information bits. The black arrow on the left represents the computing direction of the frozen bits. Since the value of the frozen bits does not affect the encoding result, we set them to zero. The final outputs of the encoding are the value of the rightmost black circles.

For a SPC encoder in Fig. 1, we can reduce the XOR operation to obtain the output of $x_{0}, x_{2}$, and $x_{4}$. From Fig. 1, we can obtain

$x_{0}=u_{0} \oplus u_{4} \oplus u_{2} \oplus u_{6} \oplus u_{1} \oplus u_{5} \oplus u_{3} \oplus u_{7}$

Consider that $u_{0}, u_{2}$, and $u_{4}$ are frozen bits which are set zero. Then, Eq. (8) can be rewritten as

$x_{0}=u_{6} \oplus u_{1} \oplus u_{5} \oplus u_{3} \oplus u_{7}$

From Fig. 1, we also have the following relations

$$
\left\{\begin{array}{l}
u_{7}=x_{7} \\
u_{6}=x_{6} \oplus x_{7} \\
u_{5}=x_{5} \oplus x_{7} \\
u_{3}=x_{3} \oplus x_{7} \\
u_{1}=x_{1} \oplus x_{3} \oplus x_{5} \oplus x_{7}
\end{array}\right.
$$

Substitute Eq. (10) into Eq. (9), $x_{0}$ becomes $x_{0}=\left(x_{6} \oplus x_{7}\right) \oplus\left(x_{1} \oplus x_{3} \oplus x_{5} \oplus x_{7}\right) \oplus\left(x_{5} \oplus x_{7}\right) \oplus\left(x_{3} \oplus x_{7}\right) \oplus x_{7}$

After applying logical XOR operations, the output results become zero if the input values are the same. Thus, Eq. (11) can be rewritten as

$x_{0}=x_{6} \oplus x_{1} \oplus x_{7}$

Similarly, $x_{2}$ and $x_{4}$ can be derived

$$
\begin{aligned}
x_{2} & =u_{2} \oplus u_{6} \oplus u_{3} \oplus u_{7}=u_{6} \oplus u_{3} \oplus u_{7} \\
& =\left(x_{6} \oplus x_{7}\right) \oplus\left(x_{3} \oplus x_{7}\right) \oplus x_{7}=x_{6} \oplus x_{3} \oplus x_{7} \\
x_{4} & =u_{4} \oplus u_{6} \oplus u_{5} \oplus u_{7}=u_{6} \oplus u_{5} \oplus u_{7} \\
& =\left(x_{6} \oplus x_{7}\right) \oplus\left(x_{5} \oplus x_{7}\right) \oplus x_{7}=x_{6} \oplus x_{5} \oplus x_{7}
\end{aligned}
$$

By combining Eqs. (12), (13), and (14), we simplify the encoding diagram illustrated in Fig. 1 to the diagram in Fig. 2.

Compared with the computation complexity of original algorithm [11], the proposed OEA reduces the computational units without increasing the memory bits. Figures 1 and 2 show the difference of the number of computing units. There are only four XOR computing units to be reduced in Fig. 1. However, 12 XOR operations are omitted in Fig. 2. $u_{1}, u_{3}, u_{5}, u_{6}$, and $u_{7}$ are intermediate variables that can be ignored. The gray nodes on the rightmost represent the information bits, and the black nodes on the leftmost represent the frozen bits. The right black nodes are unknown. The outputs of the SPC encoder are $x_{0}, x_{2}$, and $x_{4}$.

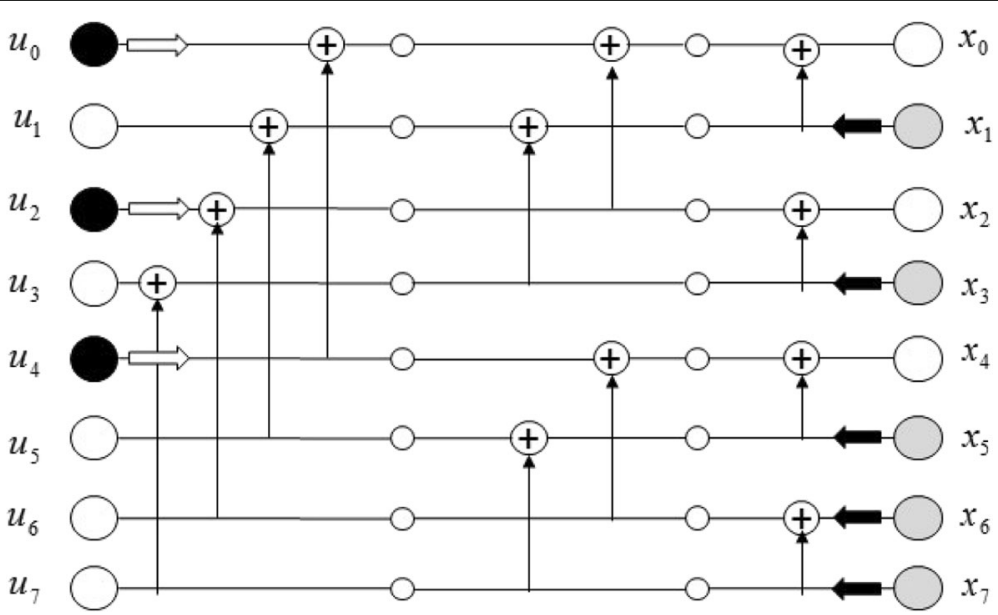

Fig. 1 SPC encoding diagram with $N=8$ and $A=\{1,3,5,6,7\}$ : The encoding direction is from bottom to top. The encoding process for information bits starts from right to left. Then, the encoding process for frozen bits is from left to right. The gray circles on the rightmost represent the information bits, and the black circles on the leftmost represent the frozen bits. The black arrow on the right represents the computing direction of the information bits. The black arrow on the left represents the computing direction of the frozen bits. The final outputs of the encoding are the value of the rightmost black circles 


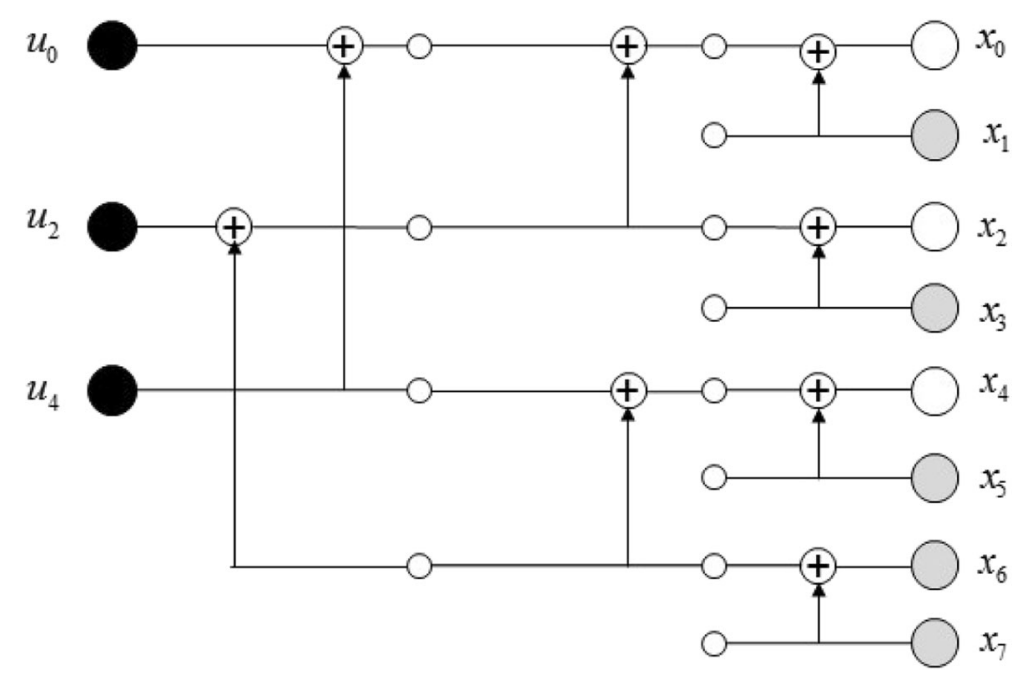

Fig. 2 Our SPC encoding optimization algorithm diagram: The gray nodes on the rightmost represent the information bits, and the black nodes on the leftmost represent the frozen bits. The right black nodes are unknown and the outputs of the SPC encoder are to obtain $x_{0}, x_{2}$, and $x_{4}$

The outputs of the SPC encoder also can be generated from a two-dimensional generator matrix. For example, we construct a generator matrix with the polar code length $N=8$

$$
G_{8}=\left[\begin{array}{llllllll}
1 & 0 & 0 & 0 & 0 & 0 & 0 & 0 \\
1 & 1 & 0 & 0 & 0 & 0 & 0 & 0 \\
1 & 0 & 1 & 0 & 0 & 0 & 0 & 0 \\
1 & 1 & 1 & 1 & 0 & 0 & 0 & 0 \\
1 & 0 & 0 & 0 & 1 & 0 & 0 & 0 \\
1 & 1 & 0 & 0 & 1 & 1 & 0 & 0 \\
1 & 0 & 1 & 0 & 1 & 0 & 1 & 0 \\
1 & 1 & 1 & 1 & 1 & 1 & 1 & 1
\end{array}\right]
$$

The codeword vector $x$ can be obtained from the matrix product of $u$ (Eq. (3)) and above $G_{8}$ (Eq. (15)). For example, we can have the element $x_{0}$ (Eq. (16)) by multiplying $u$ (Eq. (3)) with $G_{8}$ (Eq. (15))

$$
x_{0}=u_{0} \oplus u_{1} \oplus u_{2} \oplus u_{3} \oplus u_{4} \oplus u_{5} \oplus u_{6} \oplus u_{7}
$$

Comparing $x_{0}$ in Eq. (16) to $x_{0}$ in Eq. (8), we found that the encoding result is the same. One is from an SPC encoding diagram (Eq. (8)), and another one is from a generator matrix approach (Eq. (16)). The proposed OEA utilizes the characteristics of the generator matrix and the matrix transformation. For a general case with the code length $N$, Section 4 shows that the outputs from a generator matrix approach are the same as the outputs from a diagram approach. For example, when $N=8$ in Eq. (7), $A=$ $\{1,3,5,6,7\}$, and $G_{A A}{ }^{-1} G_{A A c}=[1,0,0 ; 0,1,0 ; 0,0,1$; $1,1,1 ; 1,1,1] . x_{A c}$ has the elements of $x_{0}, x_{2}$, and $x_{4}$ as outputs. These outputs are the same as the results from Eqs. (12), (13), and (14). Therefore, the characteristics of the generator matrix can be considered to use in the process of our encoding optimization algorithm for a general case.

\section{The theory of OEA}

Before discussing the characteristics of the generator matrix, we first divide the distribution of information bits [15] into two groups based on the preset value. Then, we divide the distribution of information bits into two areas based on the bit channel index. Figures 3, 4, 5, and 6 show the capacity of binary erasure channels (BEC) when the code length $N$ is $128,256,1024$, and 2048, respectively. We can map the bit channel index to the set of $A$ and the set of $A c$ according to the capacity value larger than or smaller than the preset value. For the areas of all bit channel index in $A$ or all bit channel index in $A c$, we define them as non-hybrid areas. For the area of the bit channel index belonging to both $A$ and $A c$, we define it as a hybrid area. For example, in Fig. 3, we can set up 0.7 as a preset capacity value. When the capacity value is larger than 0.7 , the bit channel index belongs to the set of $A$. When the capacity value is smaller than 0.7, the bit channel index belongs to the set of $A c$. For the set of $A$, we select the lowest index in the area with capacity value larger than 0.7 . We draw a line 1 across the lowest index and denote the left side of line 1 as a non-hybrid area. Similarly, for the set of $A c$, we select the highest index in $A c$ to the area with capacity value smaller than 0.7 . We draw a line 2 across the highest index. We call the right side of line 2 a non-hybrid area. We denote the area 


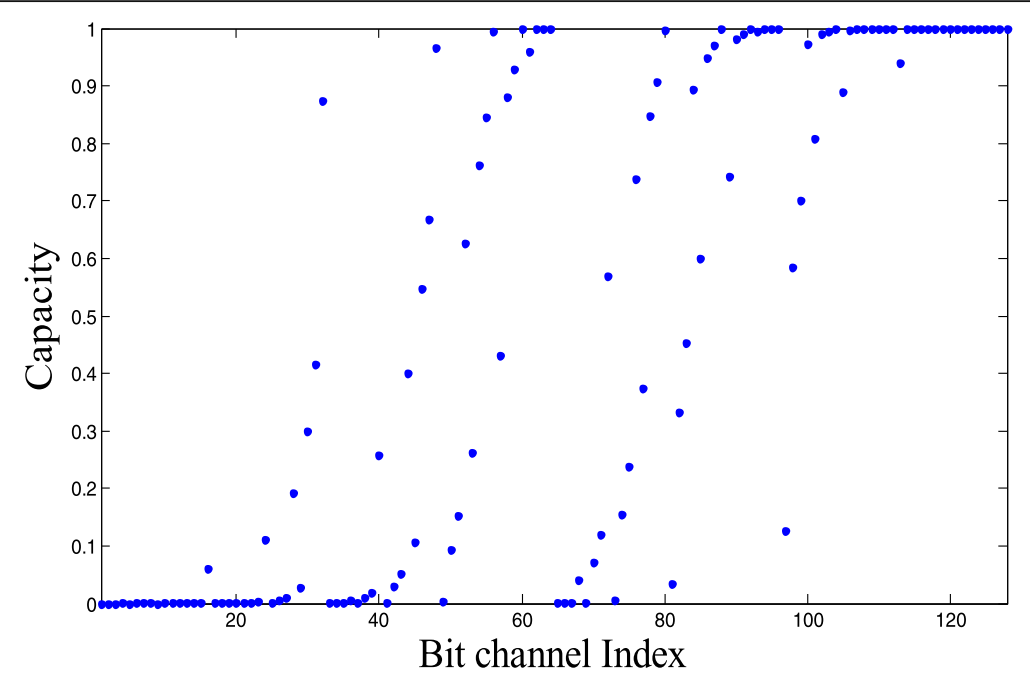

Fig. 3 The capacity of bit channel at $N=128$ : The capacity of binary erasure channels (BEC) when the code length $N$ is 128

between the line 1 and the line 2 as a hybrid area. There are both frozen bits and information bits distributed in this hybrid area. For Figs. 4, 5, and 6, we can use the same approach to divide the areas into a hybrid or a non-hybrid area and map the distribution of information bits into the set of $A$ and $A c$.

The following variables are defined to describe the highest index value in $A c$, the lowest index value in $A$, the number of information bits, and the number of frozen bits in the hybrid and non-hybrid areas. The defined variables are listed in the Table 1.

For the non-hybrid area, the index value of $p_{f i}$ represents the number of the frozen bits, and $N-p_{l f}-1$ represents the number of the information bits, where $N$ has been defined as the code length previously. The value of $N$ is the total number of all frozen bits and information bits. The width of the hybrid area in the generator matrix can be represented by $\Delta=p_{l f}-p_{f i}+1$. The value of $\Delta$ also represents the sum of the frozen bits and the information bits in this hybrid area. Figure 7 shows these defined variables of $p_{f i}, \Delta, N-p_{l f}-1$ when the code length $N$ is 16 .

In Section 2, we have denoted $G_{N}$ as a lower triangular with all ones across the diagonal and $G_{N}$ is invertible. In binary GF (2), the invertible matrix is equal to itself, $G_{N}{ }^{-1}=G_{N}$. $G_{i, j}$ represents an element in the matrix of $G_{N} . i, j \in N$, where $N=\{0,1, \ldots, N-1\}$. When $i=j, G_{i, j}=1$; when $i<j, G_{i, j}=0$.

We have following discrete function definitions. These defined functions are used to show the lower triangle

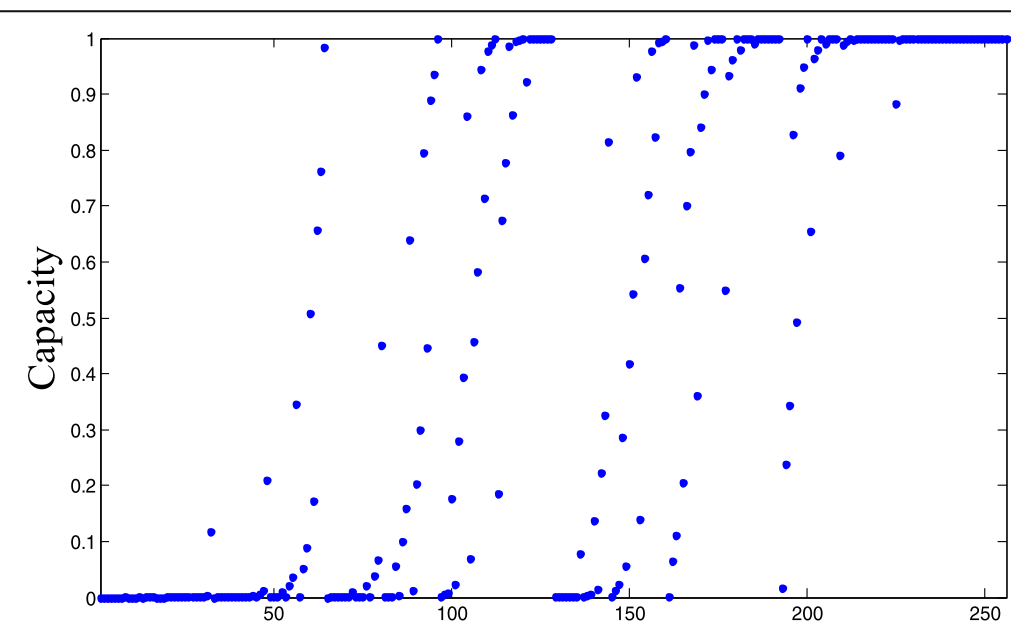

Bit channel Index

Fig. 4 The capacity of bit channel at $N=256$ : The capacity of binary erasure channels (BEC) when the code length $N$ is 256 


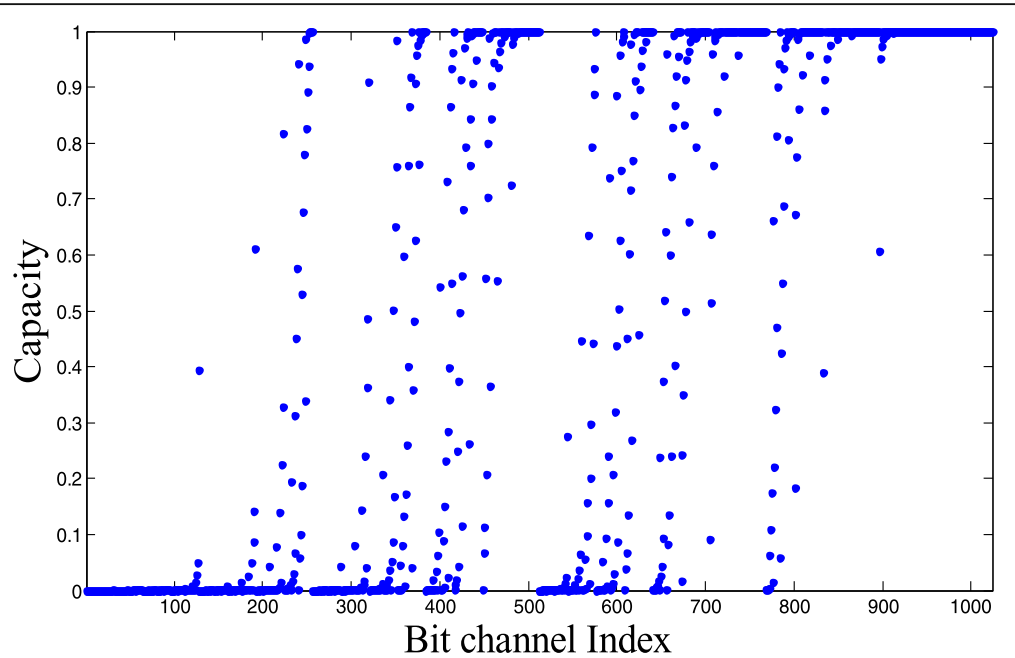

Fig. 5 The capacity of bit channel at $N=1024$ : The capacity of binary erasure channels (BEC) when the code length $N$ is 1024

structure property of the generator matrix $G_{N}$ and its submatrices of $G_{A A}$ and $G_{A A c}$.

Discrete function definition:

$f_{N}(x)=x$, where a discrete variable $x$ of the function is $x \in N$. The discrete function is $N, f_{N}(x) \subset N$.

$f_{A}(x)=A(x)$, where a discrete variable $x$ of the function is $x \in\{1,2, \ldots, K\}, K$ is the length of information bits. The discrete function is $A(x), f_{A}(x) \subset A$, and $f_{A}(1)=A(1)=p_{f i}$. $f_{A}(x)$ is a monotone increasing function.

$f_{c}(x)=A c(x)$, where the discrete variable $x$ of the function is $x \in\{1,2, \ldots, N-K\}, N$ is the code length and $N-K$ is the length of frozen bits. The discrete function is $A c(x), f_{c}(x) \subset A c$, and $f_{c}(N-K)=A c(N-K) \cdot f_{c}(x)$ is a monotone increasing function.
Since both matrix $G_{N}$ and $G_{A A}$ are square matrices, for the defined function $f_{N}(x)=x$, in which $x$ represents the $x$ th row of $G_{N}$ as well as the $x$ th column of $G_{N}, f_{N}(x)$ can represent the $f_{N}(x)$ th row of $G_{N}$ as well as the $f_{N}(x)$ th column of $G_{N}$. For $f_{A}(x)=A(x), x$ represents the $x$ th row of $G_{A A}$ and $G_{A A c}$, as well as $x$ th column of $G_{A A}$. $f_{A}(x)$ represents $A(x)$ th row in $G_{N}$ as well as $A(x)$ th column of $G_{N}$. For $f_{c}(x)=A c(x), x$ represents the $x$ th column of $G_{A A c}$ and $f_{c}(x)$ represents the $A c(x)$ th row of $G_{N}$ as well as $A c(x)$ th column of $G_{N}$.

Lemma 1: $G_{A A}$ is a lower triangular with all ones across the diagonal.

Proof: For $G_{A A}$, when $x=y, x, y \in\{1,2, \ldots, K\}, f_{A}(x)=$ $f_{A}(y)$. So $f_{N}\left(f_{A}(x)\right)=f_{N}\left(f_{A}(y)\right)$. Since $f_{A}(x), f_{A}(y) \in A \subset N$, and

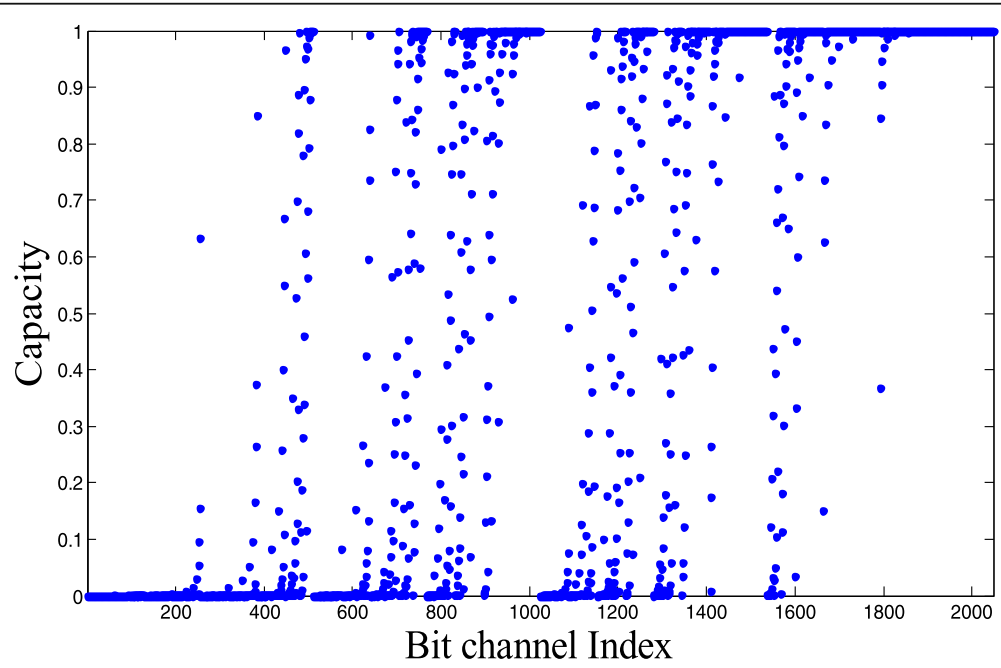

Fig. 6 The capacity of bit channel at $N=2048$ : The capacity of binary erasure channels (BEC) when the code length $N$ is 2048 
Table 1 Defined variables

\begin{tabular}{ll}
\hline Variable & Definition \\
\hline$p_{f i}$ & The first information bit index \\
$p_{\text {If }}$ & The last frozen bit index \\
$\Delta$ & The width of the hybrid area \\
$\Delta i$ & The number of information bits \\
& in hybrid area \\
$\Delta f$ & The number of frozen bits \\
& in hybrid area \\
\hline
\end{tabular}

$G_{f A(x), f A(y)}=1,\left(G_{A A}\right)_{x, y}=1$. When $x<y, x, y \in\{1,2, \ldots, K\}$, due $f_{A}(x)$ is a monotone increasing discrete function, so $f_{A}(x)<f_{A}(y)$. Hence $f_{N}\left(f_{A}(x)\right)<f_{N}\left(f_{A}(y)\right)$. Because of $f_{A}(x)$, $f_{A}(y) \in A \subset N$, we obtain that $G_{f A(x), f A(y)}=0$. Hence, $\left(G_{A A}\right)$ $x, y=0$. Given the property that the inverted matrix has the lower triangular structure if the original one has the lower triangular matrix, $G_{A A}$ is a lower triangular; therefore, the inverted $G_{A A}{ }^{-1}$ has a lower triangular matrix.

Lemma 2: A submatrix of $G_{A A c}$ has the lower triangular structure.

Proof: For $f_{A}\left(x_{1}\right)$, when $x_{1} \in\{1,2, \ldots, \Delta i\}, f_{A}\left(x_{1}\right) \in\{A(1)$, $A(2), \ldots, A(\Delta i)\}, f_{A}(1)=A(1)=p_{f i}$. For $f_{c}\left(x_{2}\right)$, in which $x_{2} \in\{N-K-\Delta f, N-K-\Delta f+1, \ldots, N-K\}, f_{c}\left(x_{2}\right) \in\{A c(N-$ $K-\Delta f), A c(N-K-\Delta f+1), \ldots, A c(N-K)\} . f_{c}(N-K)=$ $A c(N-K)=p_{l f}$. In the hybrid area, we know that $A(1)$ $<A c(N-K-\Delta f)$ and $A(\Delta i)<A c(N-K)$. As we know from the above analysis, each information bit index is smaller than the previous frozen bit index. Due to $f_{A}(x)$ and $f_{c}(x)$ are monotone increasing discrete functions, so $f_{A}\left(x_{1}\right)<f_{c}\left(x_{2}\right), G_{f A(x 1), f c(x 2)}=0$, and $\left(G_{A A c}\right)_{x 1, x 2}=0$. The property of a submatrix $G_{A A c}$ with lower triangular structure exists if $f_{A}\left(x_{1}\right)<f_{c}\left(x_{2}\right)$, where $x_{1}$ and $x_{2}$ are in different discrete sets, $x_{1} \in\{1,2, \ldots, \Delta i\}$ and $x_{2} \in\{N-K$ $\Delta f, N-K-\Delta f+1, \ldots, N-K\}$.

\section{A case study of OEA}

For the case of code length of $N=16$, suppose the code rate is $1 / 2$, we will have $K=8, A=\{3,5,7,9,11,13,14$, $15\}$, and $A c=\{0,1,2,4,6,8,10,12\}$. For the generator matrix of $G_{16}$ in Fig. 8a, the rows of information bits indices can be extracted to form $\left(G_{16}\right)_{A}$ shown in Fig. 8a, $\mathrm{b}, \mathrm{c}_{1}$, and $\mathrm{c}_{2}$ which illustrate the detailed procedures of the matrix transformation.

The row elements in the solid line box in Fig. 8a form a new matrix $\left(G_{16}\right)_{A}$ shown in Fig. $8 b$. In Fig. $8 b, f_{A}(1)=$ $A(1)=3$ and $f_{c}(N-K)=A c(N-K)=12$, so the third column represents the first information bit $p_{f i}$ and the 12 th column represents the last frozen bit $p_{l f}$. We can form $\left(G_{16}\right)_{A A}$ in Fig. $8 c_{2}$ by extracting the columns from the dashed box in Fig. 8b. To form $\left(G_{16}\right)_{A A c}$ shown in Fig. $8 c_{1}$, we can use the remaining columns from the Fig. 8 b. By the Lemma $1,\left(G_{16}\right)_{A A}$ is a lower triangular matrix with all ones across the diagonal, $\left(G_{16}\right)_{A A}{ }^{-1}=$ $\left(G_{16}\right)_{A A}, G_{A A}^{-1}$ is a lower triangular matrix. By the Lemma 2, $\left(G_{16}\right)_{A A c}$ has a lower triangular structure. For the general case of $N=2^{n}, n \geq 1$, the dimension of the lower triangular matrix in $\left(G_{16}\right)_{A A c}$ is $N-K-p_{f i}+1$. The dimension of $\left(G_{16}\right)_{A A C}$ is $N-K$, minus the first part of the matrix, so the remaining matrix size is $d=N-K$ $-p_{f i}+1 . G_{A A}^{-1}$ is the inverse of itself, and it is a lower triangular matrix with the dimension of $K$.

The matrix $\left(G_{16}\right)_{A A}{ }^{-1}$ and $\left(G_{16}\right)_{A A C}$ in Fig. 9a and b are partitioned to obtain a block of a zero submatrix. To further discuss about general case, Fig. 9a can be

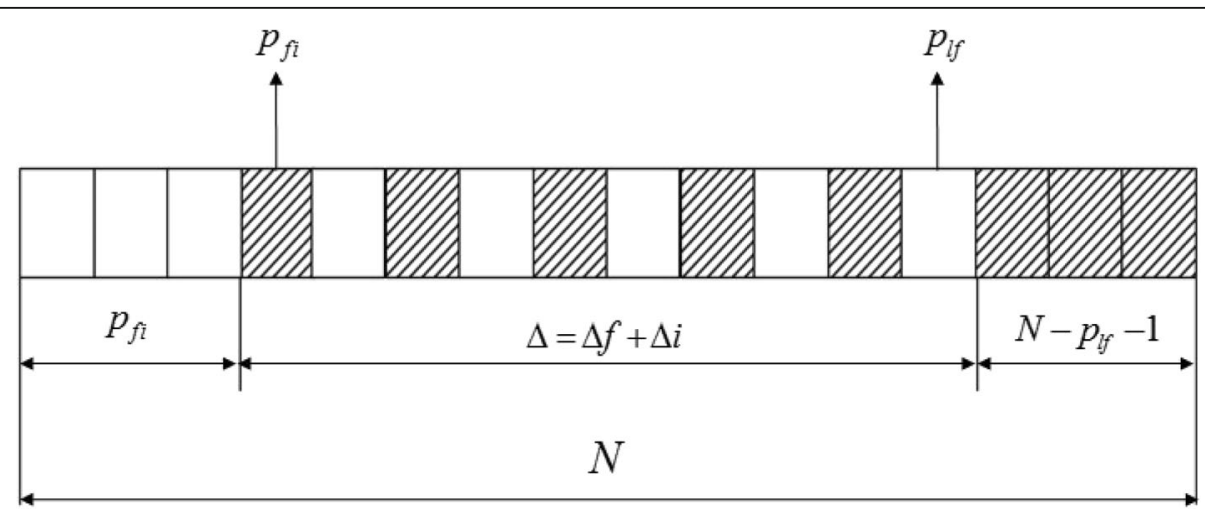

frozen bit

information bit

Fig. 7 The information bits and frozen bits distribution at $N=16$ : For the non-hybrid area, the index value of $p_{f i}$ represents the number of the frozen bits, and $N-p_{I f}-1$ represents the number of the information bits, where $N$ has been defined as the code length previously. The value of $N$ is the total number of all frozen bits and information bits. The width of the hybrid area in the generator matrix can be represented by $\Delta=p_{\text {If }}-p_{f i}+1$. The value of $\Delta$ also represents the sum of the frozen bits and the information bits in this hybrid area. These defined variables of $p_{f i} \Delta, N-p_{\text {If }}-1$ when the code length $N$ is 16 


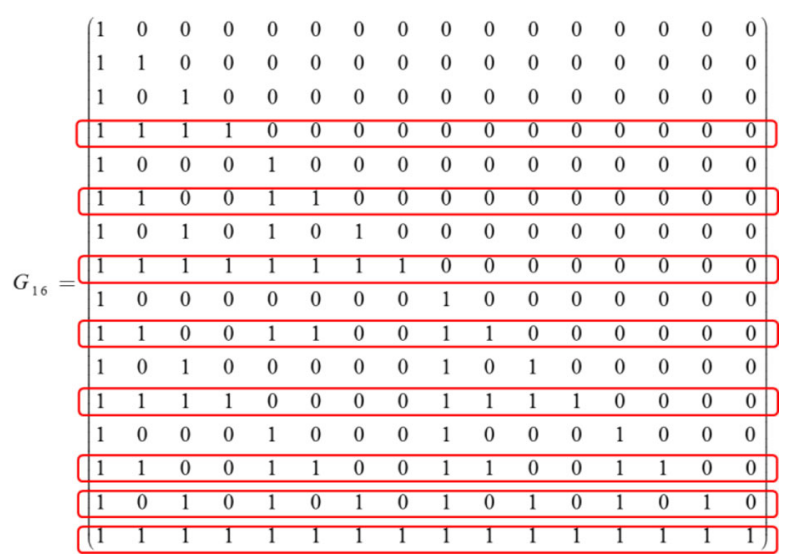

(a)

$p_{f i}+1$

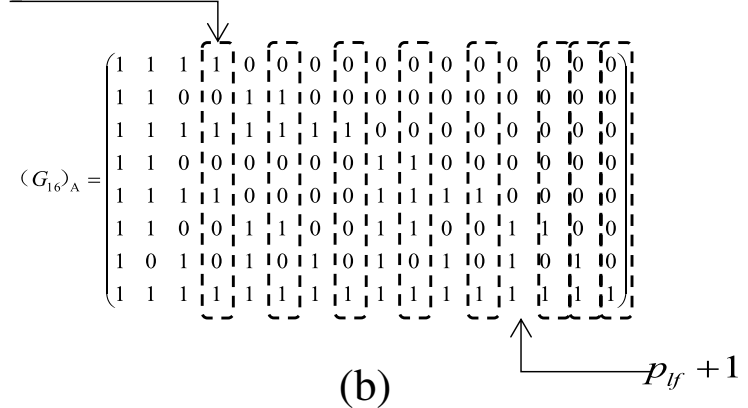

(b)

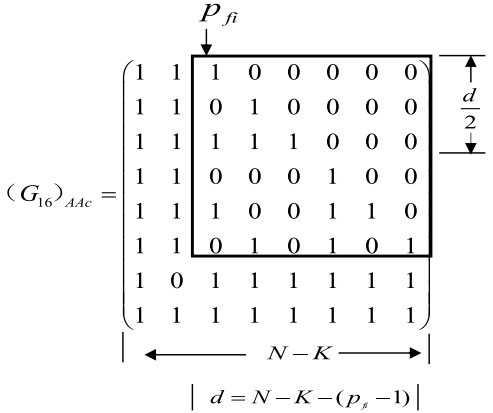

(c1)

$$
\left(G_{16}\right)_{A 4}=\left(\begin{array}{llllllll}
1 & 0 & 0 & 0 & 0 & 0 & 0 & 0 \\
0 & 1 & 0 & 0 & 0 & 0 & 0 & 0 \\
1 & 1 & 1 & 0 & 0 & 0 & 0 & 0 \\
0 & 0 & 0 & 1 & 0 & 0 & 0 & 0 \\
1 & 0 & 0 & 1 & 1 & 0 & 0 & 0 \\
0 & 1 & 0 & 1 & 0 & 1 & 0 & 0 \\
0 & 0 & 0 & 0 & 0 & 0 & 1 & 0 \\
1 & 1 & 1 & 1 & 1 & 1 & 1 & 1
\end{array}\right)
$$

(c2)

Fig. 8 Matrix extraction process: For the generator matrix of $G_{16}$ in $a$, the rows of information bits indices can be extracted to form $\left(G_{16}\right)_{A}$ shown in $\mathbf{b}$. $\mathbf{a}-\mathbf{c}_{\mathbf{2}}$ illustrate the detailed process of matrix transformation. The row elements in the solid line box in a are formed into a new matrix $\left(G_{16}\right)_{A}$ shown in $\mathbf{b}$

partitioned and written as several submatrices of $g_{1}, g_{2}$, $g_{3}$, and $g_{4}$ in Fig. 10a. The submatrix $g_{2}$ is a zero matrix. Figure $9 \mathrm{~b}$ can be partitioned into submatrices of $c_{1}, c_{2}$, $c_{3}$, and $c_{4}$ in Fig. 10b. The submatrix $c_{2}$ is a zero matrix.

\section{The block matrix in Fig. 10a and $b$ can be written as Eqs. (17) and (18), respectively}

$$
G_{A A}^{-1}=\left(\begin{array}{ll}
g_{1} & g_{2} \\
g_{3} & g_{4}
\end{array}\right)
$$

$$
G_{A A c}=\left(\begin{array}{ll}
c_{1} & c_{2} \\
c_{3} & c_{4}
\end{array}\right)
$$

When $d$ is even, the sizes of the submatrices of $g_{1}$, $g_{2}, g_{3}$, and $g_{4}$ are $\frac{d}{2} \times \frac{d}{2}, \frac{d}{2} \times\left(K-\frac{d}{2}\right),\left(K-\frac{d}{2}\right) \times \frac{d}{2}$, and $(K-$ $\left.\frac{d}{2}\right) \times\left(K-\frac{d}{2}\right)$, respectively. And the sizes of submatrices of $c_{1}, c_{2}, c_{3}$, and $c_{4}$ are $\frac{d}{2} \times\left(N-K-\frac{d}{2}\right), \frac{d}{2} \times \frac{d}{2},(K-$ $\left.\frac{d}{2}\right) \times\left(N-K-\frac{d}{2}\right)$, and $\left(K-\frac{d}{2}\right) \times \frac{d}{2}$, respectively. Due to 


$$
\left(G_{16}\right)_{A 4}{ }^{-1}=\left(\begin{array}{ccc:ccccc}
1 & 0 & 0 & 0 & 0 & 0 & 0 & 0 \\
0 & 1 & 0 & 0 & 0 & 0 & 0 & 0 \\
1 & 1 & 1 & 0 & 0 & 0 & 0 & 0 \\
\hdashline 0 & 0 & 0 & 1 & 0 & 0 & 0 & 0 \\
1 & 0 & 0 & 1 & 1 & 0 & 0 & 0 \\
0 & 1 & 0 & 1 & 0 & 1 & 0 & 0 \\
0 & 0 & 0 & 0 & 0 & 0 & 1 & 0 \\
1 & 1 & 1 & 1 & 1 & 1 & 1 & 1
\end{array}\right) .
$$

(a)

$$
\left(G_{16}\right)_{A A C}=\left(\begin{array}{ccccc:ccc}
1 & 1 & 1 & 0 & 0 & 0 & 0 & 0 \\
1 & 1 & 0 & 1 & 0 & 0 & 0 & 0 \\
1 & 1 & 1 & 1 & 1 & 0 & 0 & 0 \\
\hdashline 1 & 1 & 0 & 0 & 0 & 1 & 0 & 0 \\
1 & 1 & 1 & 0 & 0 & 1 & 1 & 0 \\
1 & 1 & 0 & 1 & 0 & 1 & 0 & 1 \\
1 & 0 & 1 & 1 & 1 & 1 & 1 & 1 \\
1 & 1 & 1 & 1 & 1 & 1 & 1 & 1
\end{array}\right) \ldots
$$

(b)

Fig. 9 Matrix segmentation at $N=16$ : The matrix $\left(G_{16}\right)_{A A}{ }^{-1}$ and $\left(G_{16}\right)_{A A C}$ in $\mathbf{a}$ and $\mathbf{b}$ can be divided to obtain the submatrix with all elements of zeros, respectively. To further discussion general case, a can be segmented into submatrices of $g_{1}, g_{2}, g_{3}$, and $g_{4}$ shown in Fig. 10 a. The submatrix $g_{2}$ is a zero matrix

the zero property of $g_{2}$ and $c_{2}$, Eq. (7) can be rewritten as

$$
x_{A c}=x_{A} G_{A A}^{-1} G_{A A c}=x_{A}\left(\begin{array}{cc}
g_{1} c_{1} & 0 \\
g_{3} c_{1}+g_{4} c_{3} & g_{4} c_{4}
\end{array}\right)
$$

For Eq. (19), when $d$ is even, the dimension of the zero submatrix in $G_{A A}{ }^{-1} G_{A A c}$ is $\frac{d}{2} \times \frac{d}{2}$; when $d$ is odd, the size of the zero submatrix in $G_{A A}{ }^{-1} G_{A A c}$ is $\frac{d-1}{2}$ $\times \frac{d-1}{2}$. The computing procedures are omitted when multiplying such zero submatrix during an encoding process. Therefore, we can save the computing resources after applying the proposed OEA. The OEA is universal for general cases. The pseudocodes of our OEA are listed in the algorithm for the proposed OEA. We can clearly understand the characteristic of the proposed algorithm and the difference between the encoding algorithm in [11].

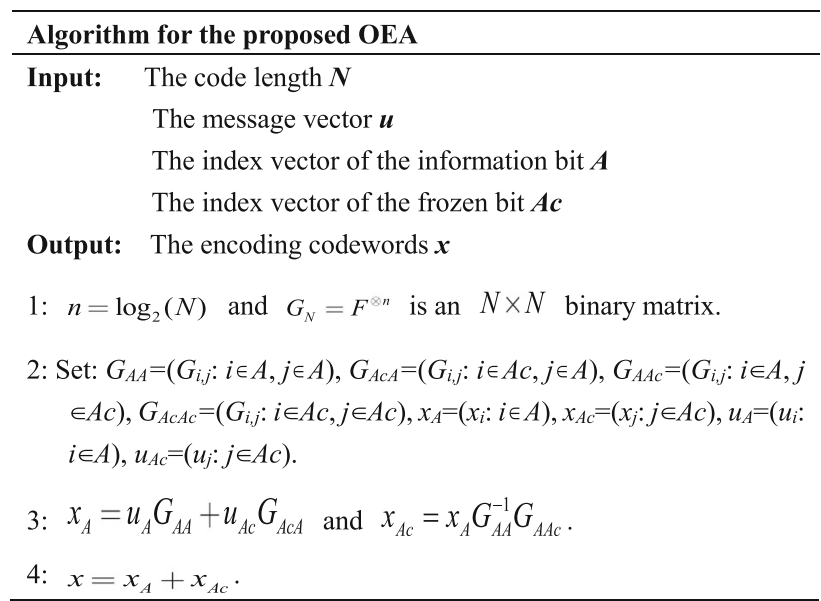

\section{Result analysis}

Table 2 discusses the dimension $d$ of lower triangular submatrix of GAAc at different code lengths of $N$ and

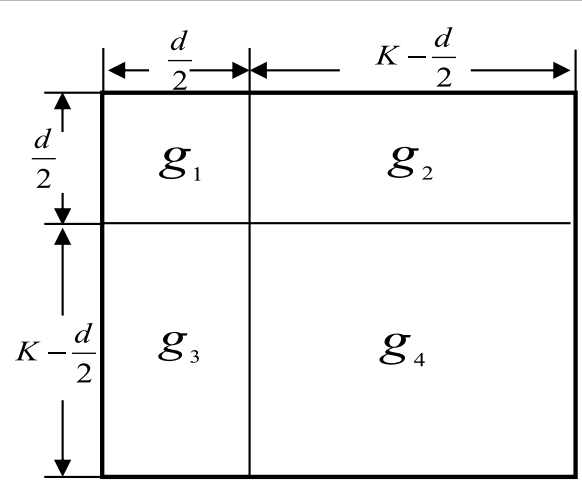

(a)

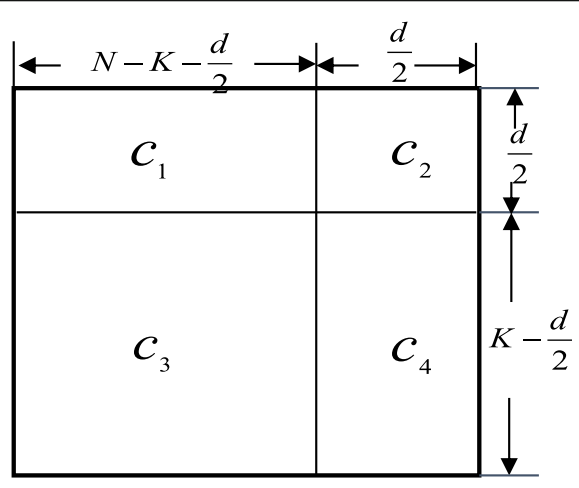

(b)

Fig. 10 Matrix segmentation at $N=2^{n}$ : Fig. 9 a can be partitioned and written as several submatrices of $g_{1}, g_{2}, g_{3}$, and $g_{4}$ shown in a. Fig. $9 b$ can segmented into submatrices of $c_{1}, c_{2}, c_{3}$, and $c_{4}$ shown in $\mathbf{b}$. The submatrix $c_{2}$ is a zero matrix 
Table 2 Detailed data of $d$

\begin{tabular}{lllll}
\hline$N$ & $R$ & $K$ & $p_{f i}$ & $d$ \\
\hline 1024 & 0.5 & 512 & 16 & 497 \\
& 0.75 & 768 & 4 & 253 \\
& 0.9 & 922 & 4 & 99 \\
2048 & 0.5 & 1024 & 16 & 1009 \\
& 0.75 & 1536 & 8 & 505 \\
& 0.9 & 1843 & 4 & 202 \\
4096 & 0.5 & 2048 & 32 & 2017 \\
& 0.75 & 3072 & 8 & 1017 \\
& 0.9 & 3686 & 1 & 410 \\
\hline
\end{tabular}

different code rates of $R$, where $d=N-K-p_{f i}+1$. Detailed data of three different code lengths and three different code rates for each of them are listed in this table.

Figure 11 shows the ordinate in a linear plot. The relation between $d$ and $R$ is in a linear relationship for each of code length $N$. The value changes of $p_{f i}$ can be negligible to a code length.

Figure 12 shows the variation trend of $d$ with the code length $N$. At different code rates (for example, $R=0.5$, 0.75 , and 0.9), $d$ increases when the code length $N$ becomes short. Especially, when $R=K / N=0.5$, the growth trend is faster than others. In other words, the smaller the code rate is, the greater the value of $d$ will be.

After applying the proposed OEA to the transformed matrix, the percentages of zero elements of the lower triangular part are shown in Table 3. For SPC and NSPC, the percentages of zero elements shown in Table 3 are calculated by Eqs. (20) and (21), respectively.

$$
P_{O E A-N S P C}=\frac{\left(d^{2}-d\right) / 2}{N^{2}}
$$

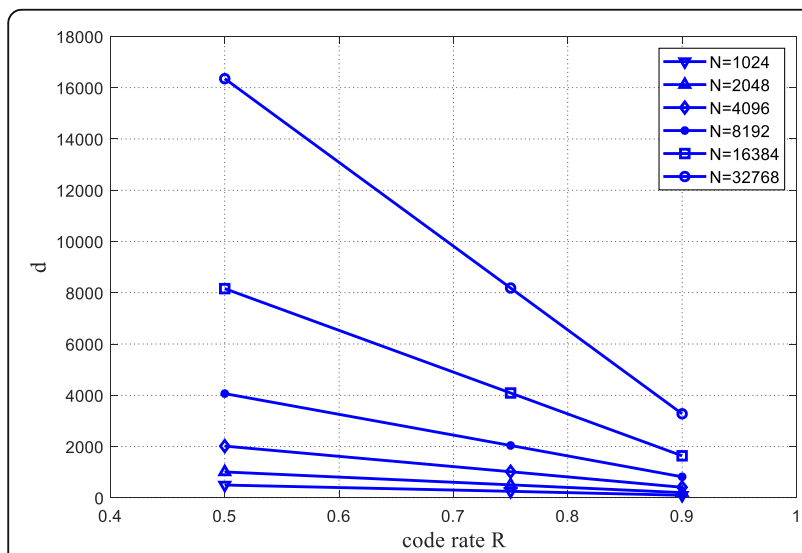

Fig. 11 Variation trend of $d$ with code rate $R$ : The ordinate in a linear form. The relation between $d$ and $R$ is in linear for each of code length $N$. The value changes of $p_{f i}$ can be negligible to a code length

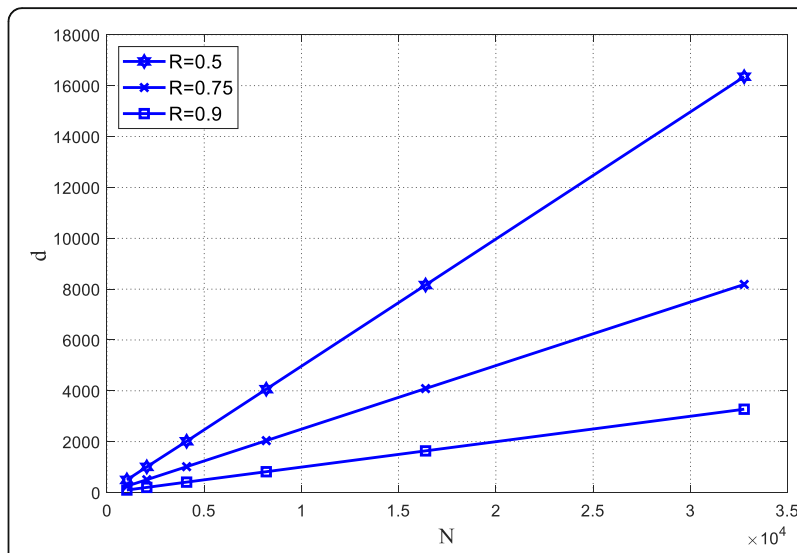

Fig. 12 Variation trend of $d$ with code length $\log _{2} N$ for a different $R$ : The variation trend of $d$ with code length $N$. And it shows a semilogarithmic relationship. At different code rates, $d$ increases with the code length $N$ for $R=0.5,0.75$, and 0.9 , respectively

$P_{O E A-S P C}=\frac{\left(d^{2}-d\right) / 2}{N \times(N-K)}$

For NSPC, $0.47 \%$ of the computing resources can be saved when $N=1024$ and $R=0.9$. However, when $N=$ 1024 and $R=0.5$, only $11.8 \%$ of the computing resources can be saved. For SPC, $58.5 \%$ of the resource consumption can be saved when code rate $R=0.5$ and the code length $N=2048$. When the code rate increases, the percentage of zero elements in the lower triangle decreases. However, the percentage is almost invariable at different code lengths for SPC and NSPC at the same code rate.

The comparison of systematic polar encoders is shown in Table 4. Unlike $[10,11]$, we use the total times of XORs to represent the computational complexity in this paper. For the Encoder A in [10] and the SPC in [11], the times of XORs can be approximated as $\left(N^{2}+N\right)\left(n^{2}\right.$ $+n) / 2>\frac{n^{2}}{2} \cdot N^{2}>N^{2}$ and $N\left(N^{2}+N\right) / 2 \geq \frac{N^{3}}{2}$, respectively. However, for the proposed OEA, the computational complexity can be decreased to $N K$ due to $N K-$ $K^{2}-N+K=(N-K)(K-1)<N K$. Therefore, the computational complexity of the OEA is lower than that of Encoder A in [10] and SPC in [11]. As for the Encoder B, Encoder C, and NSPC in [10], the times of XORs can be

Table 3 Percentages of zero elements in the transformed matrix for our OEA algorithm

\begin{tabular}{|c|c|c|c|c|c|c|}
\hline \multirow{2}{*}{$\begin{array}{l}R \\
\text { OEA }\end{array}$} & \multicolumn{2}{|c|}{$N=2^{10}=1024$} & \multicolumn{2}{|c|}{$N=2^{11}=2048$} & \multicolumn{2}{|c|}{$N=2^{12}=4096$} \\
\hline & NSPC (\%) & SPC (\%) & NSPC (\%) & SPC (\%) & NSPC (\%) & SPC (\%) \\
\hline 0.5 & 11.8 & 47.0 & 10.9 & 58.5 & 12.3 & 49.2 \\
\hline 0.75 & 3.0 & 16.2 & 3.0 & 16.2 & 3.1 & 16.4 \\
\hline 0.9 & 0.47 & 5.2 & 0.48 & 5.4 & 0.49 & 5.5 \\
\hline
\end{tabular}


Table 4 Comparison of systematic polar encoders

\begin{tabular}{lllll}
\hline Algorithm & Recursion & Memory & XORs (times) & Hardware implementation \\
\hline Encoder A [10] & No & $N\left(1+\log _{2} N\right)$ & $\left(N^{2}+N\right)\left(n^{2}+n\right) / 2$ & Yes \\
Encoder B [10] & Yes & $2 N-1$ & $N\left(1+\log _{2} N\right)$ & No \\
Encoder C [10] & Yes & $N$ & $N\left(1+2 \log _{2} N\right)$ & No \\
NSPC [10] & Yes/No & $2 N$ & $\left(N \log _{2} N\right) / 2$ & No \\
SPC [11] & No & $N$ & $N\left(N^{2}+N\right) / 2$ & Yes \\
Our OEA & No & $N$ & $N K-K^{2}-N+K$ & Yes \\
\hline
\end{tabular}

approximated as $N\left(1+\log _{2} N\right)>o(N), \quad N\left(1+2 \log _{2} N\right)>$ $o(N)$, and $\frac{N}{2} \log _{2} N>o(N)$, respectively. Compared with the recursive algorithms Encoder B, Encoder C, and NSPC in [10], the advantage of the times of XORs of the proposed OEA is not obvious, but it is beneficial to hardware implementation. For the proposed OEA, the operation of the matrix segmentation and transformation can be completed in the preprocessing stage, followed by multiplication of matrix and vector, which can be realized by XOR gate. There is no reverse transmission process of signal, which is beneficial to the timing and pipeline design of the hardware. The Encoder B, Encoder C, and NSPC in [10] are implemented by the recursive algorithm based on divide-and-conquer method in the software design, and the signal is passed from the first level to the last level, and then passed back to the first level. This process of bidirectional transmission of signals is not conducive to hardware implementation, especially in high-speed pipeline structures.

\section{Conclusions and prospect}

In this paper, we propose an optimization algorithm OEA for SPC. The number of zero elements in the transformed generator matrix and their locations can be determined in the proposed OEA. In the case of code rate reaching 0.5 , half of the number of XOR computing units can be omitted to save computation resources due to the large number of zero elements found in the submatrix. For other code rates higher than 0.5, a smaller number of the computation units is saved. The proposed OEA not only reduces the number of XOR computing units compared with the existing non-recursive algorithms, but also is beneficial to hardware implementation compared with the existing recursive algorithms.

\section{Abbreviations}

BEC: Binary erasure channels; NSPC: Non-systematic polar codes; OEA: Optimized encoding algorithm; SPC: Systematic polar codes; XOR: Exclusive Or

\section{Acknowledgements}

The authors are grateful for the valuable comments and suggestions provided by the reviewers.

\section{Authors' contributions}

$J \mathrm{~L}, \mathrm{XW}$, and ZZ performed the experiments and wrote the paper. $Y W, H C, Z \mathrm{~L}$, and LS helped revise and improve the whole paper. All authors read and approve the final manuscript.

\section{Authors' information}

Jun Li was born on February 1977. He received the Ph.D degree in Communication and Information System from South China University of Technology, Guangzhou, China, in 2005. He worked in ZTE Corporation in 2005. And he has been an associate professor since 2007, and now he is in Binjiang College, Nanjing University of Information Science and Technology. His research interests include channel coding, signal detection, LTE physical layer standard, and wireless resource optimization.

Zhihong Zhang is a Master's student in College of Information Engineering in China Jiliang University. She received the B.E. degree in Communication Engineering from Yantai University, in 2015. Her research interests include signal and information processing.

Xiumin Wang was born in April 1, 1963. She received the B.E. degree in Communication and Electronic System from Dalian University of Technology. She is now a professor, Associate Dean of College of Information Engineering in China Jiliang University. Her research interests include signal and information processing.

Prof. Yu Wang earned her Ph.D. degree in Electrical Engineering from the Graduate Center of the City University of New York. Dr. Wang joined the Computer Engineering Technology Department of New York City College of Technology in 2009. Her primary area of interest includes LTE networks, realtime systems, network protocols, and embedded systems.

Haiyan Cao received the Ph.D degree in information engineering from South China University of Technology, Guangzhou, China, in 2006. She is currently an associate professor of College of Communication Engineering in Hangzhou Dianzi University. Her research interests include Massive MIMO, OFDM, LDPC codes, and resource allocation.

Zhengquan Li received the B.S. degree from the Jilin University of Technology in 1998, the M.S. degree from the University of Shanghai for Science and Technology in 2000, and the Ph.D. degree in circuit and system from Shanghai Jiaotong University in 2003. He is currently a Professor with Jiangnan University. He is also a Postdoctoral researcher with the National Mobile Communications Research Laboratory, Southeast University. His current research interests include space time coding and cooperative communications and massive MIMO.

Liang Shan received the B.S. degree from SouthEast University of Radio Engineering in 2001, the M.S. degree from South East University of Signal and Information processing in 2004. She is currently an associate professor with China Jiliang University. Her current research interests include signal processing and measurement.

\section{Funding}

This work was supported in part by the National Natural Science Foundation of China, under Grant No. 51874264, 61571108, 61501158, and 61379027; in part by the Open Foundation of State key Laboratory of Networking and Switching Technology, Beijing University of Posts and Telecommunications, under Grant SKLNST-2016-2-14; in part by the Jiangsu Postdoctoral Foundation, under Grant No. $1302059 B$

Competing interests

The authors declare that they have no competing interests. 


\section{Author details}

${ }^{1}$ College of Information Engineering, China Jiliang University, Hangzhou 310018, China. ${ }^{2}$ Binjiang College Nanjing University of Information Science and Technology, Wuxi 214105, China. ${ }^{3}$ Department of computer engineering technology, New York City College of Technology, New York 11201, USA. ${ }^{4}$ College of Communication Engineering, Hangzhou Dianzi University, Hangzhou 310018, China. ${ }^{5}$ School of loT Engineering, Jiangnan University, Wuxi 214122, China.

Received: 20 June 2018 Accepted: 10 June 2019

Published online: 01 August 2019

\section{References}

1. E. Arikan, Channel Polarization: A method for constructing capacity achieving codes for symmetric Serbian-input memoryless channels. IEEE Trans. Inf. Theory 55, 3051-3073 (2009)

2. E. Arikan, Channel polarization: A method for constructing capacityachieving codes, in IEEE International Symposium on Information Theory (Toronto), pp. 1173-1177 (2008).

3. S. Zhao, P. Shi and B. Wang, Designs of Bhattacharyya Parameter in the Construction of Polar Codes, in 7th International Conference on Wireless Communications, Networking and Mobile Computing (Wuhan), pp. 1-4 (2011).

4. R. Mori, T. Tanaka, Performance of polar codes with the construction using density evolution, IEEE Commun. Lett. vol. 13, no. 7, pp. 519-521 (2009)

5. A. Arpure, S. Gugulothu, FPGA implementation of polar codes based encoder architecture, in International Conference on Communication and Signal Processing (India), April 6-8, pp. 0691-0695 (2016)

6. X. Shih, P. Huang and Y. Chen, High-speed low-area-cost VLSI design of polar codes encoder architecture using radix-k processing engines, in IEEE 5th Global Conference on Consumer Electronics (Kyoto), pp. 1-2 (2016)

7. C. Zhang, J. Yang, X. You and S. Xu, Pipelined implementations of polar encoder and feed-back part for SC polar decoder, in IEEE International Symposium on Circuits and Systems (Lisbon), pp. 3032-3035 (2015)

8. H. Yoo, I.-C. Park, Partially parallel encoder architecture for long polar codes. IEEE Transactions on Circuits and Systems II: Express Briefs 62(3), 306-310 (2015)

9. E. Arikan, Systematic polar coding. IEEE Commun Lett 15(8), 860-862 (2011)

10. H. Vangala, Y. Hong, E. Viterbo, Efficient algorithms for systematic polar encoding. IEEE Commun Lett 20(1), 17-20 (2016)

11. G. TaiChen, Z. Zhaoyang, Z. Caijun, Z. Liang, A low complexity encoding algorithm for systematic polar codes. IEEE Commun Lett 20(7), 1277-1280 (2016)

12. G. Sarkis, P. Giard, A. Vardy, C. Thibeault, WJ. Gross, Fast polar decoders: Algorithm and implementation. IEEE J Sel Areas Commun 32(5), 946-957 (2014)

13. G. Sarkis, I. Tal, P. Giard, A. Vardy, Flexible and low-complexity encoding and decoding of systematic polar codes. IEEE Trans Commun 64(7), 2732-2745 (2016)

14. X. Wang, T. Ge, J. Li, C. Su, F. Hong, Efficient multi-rate encoder of QC-LDPC codes based on FPGA for WIMAX standard. Chinese Journal of Electronics 26(2), 250-255 (2017)

15. X. Wang, W. Cao, J. Li, et al., Improved min-sum algorithm based on density evolution for low-density parity check codes. IET communications 11(10), 1582-1586 (2017)

16. X. Wang, J. Li, Y. Wang, et al., Design of dual-mode decoder based on LDPC/turbo code. IET communications 11(8), 1325-1329 (2017)

17. H. Vangala, E. Viterbo, Y. Hong, A comparative study of polar code constructions for the AWGN channel. Mathematics 1, 1-7 (2015)

\section{Publisher's Note}

Springer Nature remains neutral with regard to jurisdictional claims in published maps and institutional affiliations.

\section{Submit your manuscript to a SpringerOpen ${ }^{\odot}$ journal and benefit from:}

- Convenient online submission

- Rigorous peer review

- Open access: articles freely available online

- High visibility within the field

- Retaining the copyright to your article

Submit your next manuscript at $\boldsymbol{\nabla}$ springeropen.com 